\title{
The role of the hippocampus in the performance of a continuous nonmatching task
}

\author{
J. K. FOSTER and J. N. P. RAWLINS \\ University of Oxford, Oxford, England
}

\begin{abstract}
Rats with hippocampal aspiration lesions (HIPP), cortical control lesions (CORT), or sham operations (SHAM) were trained on a continuous nonmatching task, using a bright or a dim wall light as the stimulus. Trials with the bright light were intermixed with trials with the dim light. On trials when the stimulus had changed, leverpressing was reinforced (nonmatch trials). When a stimulus was repeated, leverpressing was not reinforced (match trials). The intertrial intervals were varied and between-trial interference was introduced in order to compare the differential effects of these manipulations across lesion groups. Performance was assessed using signal detection theory. HIPP rats showed a significantly greater tendency to respond (bias) than did SHAM animals, with CORT animals showing an intermediate level of response output. However, there were no consistent differences between lesion groups in their ability to discriminate nonmatch from match trials (sensitivity). These findings contrast with deficits in performance reported following lesions of the fornix-fimbria and hippocampus on a similar matching-to-sample task. It is suggested that differences in response requirement between the tasks may induce rats to adopt different response strategies, which may in part explain the discrepant results. A methodological note of caution is also sounded concerning the utility of computed measures of performance on operant alternation tasks. In addition to derived measures, such as sensitivity and bias, computationally simpler indices of performance should be examined: if derived measures alone are used, spurious significant differences may arise that are not born out by more detailed analyses of response and latency data.
\end{abstract}

The role of the hippocampus in nonspatial working memory has been studied extensively in monkeys and, to a lesser extent, and more latterly, in rats. The task most frequently used in primate studies is a delayed nonmatching-to-sample one that uses "junk objects." Early studies using this task suggested that combined damage to both the hippocampus and the amygdala was required to produce an enduring impairment in recognition memory (Mishkin, 1982). However, more recent evidence from primates suggests that damage to the hippocampal structures alone may produce a profound nonspatial memory impairment (Squire \& Zola-Morgan, 1991).

In rats, on the other hand, there has, until recently, been a heavy reliance on spatial memory testing, on which hippocampal rats show reliable impairments ( $O$ 'Keefe \& Nadel, 1978; Rawlins, 1985). In a nonspatial task, lesions combining damage to the hippocampus and amygdala profoundly impaired choice accuracy in a nonmatching-tosample object recognition task using trial-unique stimuli

This research was supported by a grant from the United Kingdom Medical Research Council. J.K.F. held a studentship from the United Kingdom Science and Engineering Research Council. We would like to thank Sara Fearn for her histological assistance and Jeremy Broad for his help with the photography. We are also grateful for Paul Heatlie's assistance with the surgery and for the comments of anonymous referees on earlier versions of the manuscript. Correspondence should be addressed to J. K. Foster, Rotman Research Institute of Baycrest Centre, 3560 Bathurst Street, North York, ON, Canada M6A 2EI. when a delay was imposed (Aggleton, Blindt, \& Rawlins, 1989), whereas bilateral hippocampal aspiration lesions did not reduce response accuracy relative to control operations on this task (Aggleton, Hunt, \& Rawlins, 1986). Hippocampectomy also left animals capable of showing good list learning on the same task (K. Steele, personal communication, 1988). However, the rats that could perform the object nonmatching task well also showed a profound and enduring impairment on a similar spatial working memory task (Aggleton et al., 1986).

In contrast to the findings obtained by Aggleton et al. (1986) on a nonspatial recognition task, Raffaele and Olton (1988) observed an impairment in choice accuracy on a similar nonspatial delayed matching-to-sample task with complete fornix-fimbria lesions alone, using repeated stimuli throughout testing. Furthermore, work in this laboratory has recently shown that when the same two "junk goalboxes" (similar to the stimuli used by Aggleton et al., 1986, 1989) are used throughout the day's test session, and the working memory task is changed to a matchingto-sample rule, then both hippocampal and fornix lesions produce a severe impairment in acquisition (Rawlins, personal communication; Rawlins, Lyford, \& Seferiades, 1991). This contrasts with findings obtained using the standard object nonmatching procedure, in which each goalbox should be chosen only once in each day's session (and intrasession interference is therefore low), in which no impairment was observed by Rawlins et al. following hippocampectomy. It therefore appears as if there 
may be an interaction between the level of within-session interference and the extent of hippocampal-amygdalar damage in determining the effect of a particular lesion on nonspatial task performance.

The experiment reported here was designed to investigate further the involvement of the hippocampus in nonspatial working memory. Rats were trained to perform the continuous nonmatching-to-sample (CNM) task designed by Pontecorvo (1983). In the CNM task, a variable number of trials with one of two possible stimuli (bright and dim wall lights) alternates with a variable number of trials with the other stimulus. The first response during nonmatch trials, in which the stimulus differs from that presented on the previous trial, is reinforced. Responses on match trials, in which the stimulus is the same as that presented on the previous trial, are not reinforced. The subject therefore has to remember the stimulus presented on the previous trial and compare it with the stimulus on the present trial in order to determine whether a response will be reinforced. Retention can be inferred from the trial response accuracy, and memory decay can be assessed by determining the relationship between accuracy and the interval between successive stimulus presentations.

The CNM task shares features of both Aggleton et al.'s $(1986,1989)$ object recognition nonmatch task and the matching-to-sample task used by Raffaele and Olton (1988). In common with Aggleton et al.'s task, to perform well, subjects are required to respond selectively to a stimulus that differs from that which was presented previously; in common with the task used by Raffaele and Olton, only two stimuli are employed throughout testing, so the level of within-session proactive interference should be high.

Subjects' scores on the task were assessed using signal detection theory (SDT; Green \& Swets, 1966). SDT assumes that performance on a yes/no discrimination task depends upon two independent factors: the subject's ability to detect the appropriate stimulus for a response (sensitivity) and the subject's general preference for responding (bias). Equations derived from SDT enable one to compute individual intrasession scores of sensitivity and bias from the probability that the subject will make correct (hit) and incorrect (false alarm) responses.

If hippocampectomy decreases behavioral inhibition, as claimed by some theorists (Douglas, 1967; Gray, 1982; Kimble, 1968), it should increase the overall tendency to respond, and thus cause a relative increase in bias, but have little or no effect on sensitivity. However, if removal of the hippocampus impairs working memory (Olton, Becker, \& Handelmann, 1979) or the association of temporally discontiguous events (Rawlins, 1985), it should reduce the accuracy of responding (sensitivity), as in Raffaele and Olton's (1988) matching-to-sample task, but it should have little or no effect on bias. According to the temporal discontiguity model, the extent of the impairment in sensitivity should further increase in proportion to the length of the intertrial delay. Alternatively, if removal of both the hippocampus and the amygdala is necessary to cause a serious impairment in memory for nonspatial stimuli, as observed in Aggleton et al.'s (1986, 1989) object nonmatching experiments, then removal of the hippocampus alone should have little or no effect on sensitivity.

In the present experiment, performance on the $\mathrm{CNM}$ task was therefore compared with the predictions of each of these theories. In the first stage, the procedure was similar to that originally used by Pontecorvo (1983). After rats had achieved a stable level of performance on a continuous reinforcement (CRF) schedule, they were introduced to the CNM procedure in order to examine whether the task would discriminate between different lesion groups. In subsequent stages of the experiment, it was intended to modify the parameters by, for example, introducing intertrial interference in order to investigate in further detail the precise role of the hippocampus in performing the CNM task.

\section{METHOD}

\section{Subjects}

The subjects were 24 naive, adult, male Sprague-Dawley rats, initially weighing 248-297 g, obtained from Oxfordshire Laboratory Animal Colonies. The animals were caged individually in a temperature- $\left(22^{\circ}\right)$ and humidity-controlled (45\%-50\%) room, on a 12-h light:dark cycle (lights on at 0800 ). Food deprivation consisted of a single ration, measured by volume (approximately $22 \mathrm{~g}$ ), of Grain Harvesters food pellets, given at $1630 \mathrm{~h}$ each day, so as to maintain the animals at a near-constant weight throughout testing.

\section{Surgery}

There were three surgical treatment groups: hippocampal aspiration lesions (HIPP, $n=12$ ); cortical control lesions (CORT, $n=$ 6 ); and sham-operated control lesions (SHAM, $n=6$ ). The operations were carried out under anesthesia, using a mixture of chloral hydrate $(42 \mathrm{mg} / \mathrm{ml})$ and pentobarbitone $(9.7 \mathrm{mg} / \mathrm{ml})$, at a dose of $3 \mathrm{ml} / \mathrm{kg}$. Before operating, each rat was given an injection of $0.01 \mathrm{ml}$ vitamin $\mathrm{K}$ to prevent excessive bleeding during surgery.

Each rat was placed in a Kopf stereotaxic headholder, and the scalp was reflected to expose the skull. A hole was then made in the skull overlying the parietal cortex, and the animal was placed in a special headholder (Rawlins \& Bennett, 1980). For the HIPP rats, a portion of the dorsal occipitoparietal cortex and, as far as possible, the entire underlying hippocampus were then removed bilaterally, using aspiration under visual control. For the CORT rats, a portion of the dorsal occipitoparietal cortex overlying the hippocampus and the corpus callosum were removed to allow visualization of the alveus layer of the dorsal hippocampus. The SHAM rats received no further surgical intervention beyond the removal of the area of skull overlying the parietal cortex. For each animal, sulphonamide powder was then applied topically to the wound, the scalp was sutured and the animal was allowed to recover.

Behavioral testing commenced 13 weeks after surgery and occurred daily between 0900 and $1300 \mathrm{~h}$, with each animal being tested at approximately the same time every day on a 7-day/week testing schedule.

\section{Apparatus}

Each rat was allocated to one of eight Campden Instruments CI 410 operant test chambers, which were controlled by a NOVA minicomputer, programmed in ACT-N (Millenson, 1971, 1975). Each $24 \times 22 \times 20 \mathrm{~cm}$ box was enclosed in a light- and sound-attenuating 
chamber, which provided masking noise from an extractor fan and was enclosed in a darkened, quiet room. Within each box, a single, fixed aluminum lever was located to the left of the magazine. Illumination was provided by a 2.8 -W bulb situated above the lever, covered by a translucent lens, which produced either a dim or a bright light. The intensity ratio of the stimuli (dim/bright) was approximately 0.06 . No other illumination was present during the session. Reinforcement consisted of 3-sec access to a dipper of $11 \%$ sucrose solution $(w / v)$.

\section{Procedure}

Days 1-10: Handling. The rats were handled in groups of 4 for 10 min each day.

Days 11-20: Shaping. All rats were trained to press the lever on a CRF schedule to a criterion of 100 responses per session. On the first day of shaping, the magazine flap was secured open, free reinforcers were delivered every minute, and every press of the lever was reinforced. During the next 9 days, the magazine flap was left to move freely, the density of free reinforcers was gradually reduced, and leverpresses continued to be reinforced. Training sessions lasted $15 \mathrm{~min}$. In the final stage of shaping, reinforcers were obtained by leverpressing alone (CRF). When an animal had made 100 responses or when $15 \mathrm{~min}$ had elapsed, the session was terminated. CRF training was repeated until all animals had reached the response criterion.

Days 21-56: Stage 1. The next day, the animals were placed on the first CNM schedule. A variable number of trials with the bright wall light was alternated with a variable number of trials with the dim wall light. The first leverpress on a trial following a change in stimulus (i.e., the first leverpress on a nonmatch trial) terminated the trial and was reinforced by sucrose delivery. Leverpresses produced in response to repeated stimuli (i.e., leverpresses on match trials) were not reinforced, did not terminate the trial, and caused the match trial stimulus to be presented for a further period equal to the maximum trial duration (a correction trial), or until the animal responded once more, thus instituting a further correction trial. During correction trials, the light was therefore presented continuously until the animal refrained from responding for $10 \mathrm{sec}$. Because correction trials were repeated until the maximum trial interval elapsed without the animal making a response, animals that responded more on a match trial, and subsequent correction trials, received more exposure to the stimulus. The contingencies on a correction trial were identical to those on an initial match trial. Failure to respond on match, nonmatch, or correction trials had no scheduled consequence.

There was a total of 200 trials per session with, on average, 50 nonmatch trials and, not counting correction trials, 150 match trials per session. Each stimulus (bright or dim wall light) had an equal probability of presentation, so as to appear on approximately half of the match trials and half of the nonmatch trials. There were three different retention intervals (RIs; $1,2.5$, and $10 \mathrm{sec}$ ), each of which was scheduled within a single test session. The RIs were selected randomly, but the selection was weighted in the ratio $2: 1: 1$, so that, on average, there were $1001-\mathrm{sec}, 502.5-\mathrm{sec}$, and $5010-\mathrm{sec}$ RIs per session. (This weighting in favor of the shortest RI was used in order to encourage responding on the CNM task.) There were approximately equal proportions of the three different RIs on match trials and on nonmatch trials. A pretrial delay contingency was in effect during the last $1 \mathrm{sec}$ of the RI: if the animal responded during the pretrial delay, the onset of the next trial was postponed for an additional $1 \mathrm{sec}$. The latter contingency was included in order to prevent response bursts that were initiated during the RI from being counted as bona fide responses during the trial that followed and in order to suppress inappropriate between-trial responding. The animals from each of the three lesion groups were given the same amount of testing on the CNM task-that is, 200 trials per session (plus correction trials) for 36 sessions.
In further stages of the experiment, the task parameters were varied in order to investigate further the involvement of the hippocampus in the performance of the CNM task.

Days 57-80: Stage 2. In Stage 2, the maximum trial duration was reduced to $5 \mathrm{sec}$. There was an equal probability of occurrence of each of the three RIs in Stage 2, because all animals had achieved a reliable rate of responding on the CNM task by this stage.

Days 81-92: Stage 3. In the third stage of the experiment, the minimum RI was increased to $2.5 \mathrm{sec}$, the intermediate RI was increased to $10 \mathrm{sec}$ and the maximum $\mathrm{RI}$ was increased to $20 \mathrm{sec}$.

Days 93-120: Stage 4. In the fourth stage of the experiment, between-trial interference was introduced. A free dipper of sucrose was presented for a duration of $1 \mathrm{sec}$ after $0.5 \mathrm{sec}$ of each RI had elapsed.

\section{Histology}

At the end of behavioral testing, all surviving rats participated in a subsequent experiment, in which all CORT rats and approximately half of the HIPP rats had microinjections of ibotenic acid aimed at the amygdalar nuclei, and, following recovery from surgery, the animals continued to be tested on the CNM task. Following this experiment, the surviving animals also participated in further experiments using the same CNM task, in which the effects of administration of the muscarinic cholinergic antagonist scopolamine (hydrobromide and methyl bromide salts) and the cholinergic agonist nicotine (hydrogen tartrate salt) were investigated.

At the end of these pharmacological studies, the HIIP and CORT rats were deeply anesthetized and perfused. The brains were then removed and prepared for histological examination. For further details of the histological procedures used in these animals, see Foster and Rawlins (1992).

\section{Data Collection and Analysis}

On match trials, the data recorded were as follows: the number of match trials in which a bright stimulus was presented following the presentation of a bright stimulus on the previous trial (match bright trials), the number of these trials in which the animal responded (match bright false alarms), the sum of the latencies to respond on match bright trials (match bright false alarm total latency), the number of times in which a match bright trial was repeated because the animal made a response during the preceding match bright trial (match bright correction trials), and the number of match bright correction trials in which the animal responded (match bright repeat false alarms).

These data were recorded separately for each different RI. Similar data were recorded on match trials in which a dim stimulus was presented following the presentation of a dim stimulus on the previous trial (a match dim trial): the number of match dim trials, the number of match dim false alarms, the match dim false alarm total latency, the number of match dim correction trials, and the number of match dim repeat false alarms.

On nonmatch trials, the following data were recorded: the number of nonmatch trials in which a bright stimulus was presented following the presentation of a dim stimulus on the previous trial (nonmatch bright trials), the number of these trials in which the animal responded (nonmatch bright hits) and the sum of the latencies to respond on nonmatch bright trials (nonmatch bright hit total latency).

These data were again recorded separately for each different RI. Similar data were recorded on nonmatch trials in which a dim stimulus was presented following the presentation of a bright stimulus on the previous trial (a nonmatch dim trial): the number of nonmatch dim trials, the number of nonmatch dim hits, and the nonmatch dim hit total latency.

The number of responses each animal made in the pretrial delay period was also recorded. 
From these data, the following measures of performance were calculated per session for each rat at each of the three RIs: the probability of a hit, $p$ (hit), by taking the mean of the proportion of nonmatch bright hits and the proportion of nonmatch dim hits; the probability of a false alarm, $p(\mathrm{fa})$, by taking the mean of the proportion of match bright false alarms and the proportion of match dim false alarms; the mean hit latency, by first calculating the mean latency to respond on nonmatch bright and nonmatch dim trials and then calculating the mean of these latencies; the mean false alarm latency, by performing similar calculations on the mean latency to respond on match bright and match dim trials; the probability of repeat false alarms, $p(\mathrm{rfa})$, by taking the mean of the proportions of match bright and match dim repeat false alarms. The mean number of pretrial responses made per trial was also calculated.

The scores of $p$ (hit) and $p(\mathrm{fa})$ were then used to compute indices of sensitivity and bias. Since assumptions required for the calculation of parametric SDT indices could not be verified, nonparametric indices of sensitivity $\left(A^{\prime}\right)$ and bias $\left(B^{\prime \prime}\right)$ were computed according to the formulas given by Grier (1971) (see Appendix). $A^{\prime}$ varies between 0 and 1 , with a value of 0.5 representing chance responding and a value of 1 signifying perfect accuracy. The computed value of $B^{\prime \prime}$ can vary between -1 and 1 , with a negative value signifying a bias to respond and a positive value indicating a bias against responding. However, in the present experiment, the value of $B^{\prime \prime}$ never rose above 0 . To facilitate exposition of the data, we therefore took the modulus of $B^{\prime \prime}$ as our measure of bias, with a value of 0 representing low response bias and a value of 1 representing high response bias.

All performance measures were calculated using the SAS statistical package. Analyses of variance (ANOVAs) were performed on these measures using the SAS GLM procedure of SAS and the $2 \mathrm{~V}$ procedure of the BMDP statistical package. These programs were implemented on a VAX mainframe computer and on a 386 desktop machine, respectively. GLM was utilized to perform ANOVAs on the measures of performance detailed above. SAS was further utilized to sort and pool the principal $A^{\prime}$ and $B^{\prime \prime}$ measures. This sort was conducted in order that RI could be included as a further within-subject factor in the ANOVAs, which were subsequently performed on these measures using the BMDP $2 \mathrm{~V}$ procedure. Duncan post hoc analyses were performed to investigate significant effects $(\alpha=0.05)$. Data were analyzed in 4-day blocks. For the purpose of the analyses, trials were treated the same whether or not pretrial responding had occurred.

\section{RESULTS}

\section{Histology}

Detailed descriptions and reconstructions of cortical and hippocampal lesions of the kind used in this experiment have been previously presented (Rawlins, Feldon, \& Gray, 1980). In assessing the extent of hippocampal and cortical damage in the present experiment, a similar approach was adopted.

All HIPP rats had extensive hippocampal lesions, including damage to the dentate gyrus, the CA fields and the subiculum, but not generally to the entorhinal cortices. In all cases, most of the hippocampus was removed; in addition, the fimbria and fornix were either completely sectioned or displayed dense gliosis. Only the most caudal and ventral portions of the hippocampus were spared, and, in some cases, even these regions were damaged. Thus, only the subiculum showed consistent sparing.

A representative hippocampal lesion is illustrated in Figure 1 . It can be seen that the hippocampal damage ex- tends from close to the adseptal pole to well past the hippocampal flexure. All rats had total or near total ablations of the hippocampus at the anterior plane of assessment (corresponding to Plate 38 in König \& Klippel's, 1963, atlas). At the intermediate plane of assessment, most rats had sustained total ablation of the hippocampus (corresponding to Plate 45 in König \& Klippel's atlas). In all rats, the damage extended into the posterior plane of assessment (corresponding to Plate 50 in König \& Klippel's atlas), although, at this level, the damage was quite variable, ranging from total destruction to rather restricted lesions. The extrahippocampal damage in the HIPP group was similar to that reported earlier (Rawlins et al., 1980), in terms of apparent gliosis and scarring in the thalamus. This seemed to stem from non-specific damage, possibly mediated by pressure during or following surgery. None of these patches was large enough to warrant exclusion from the study.

All rats in the CORT group had sustained bilateral damage, which extended through the neocortex and the corpus callosum to expose the alveus of the dorsal hippocampus (Figure 1). The extent of tissue destruction was comparable to the neocortical damage sustained in the HIIPP group, thus serving as a control for the inevitable extrahippocampal damage produced when aspirating the hippocampus, rather than as a nonspecific control for the volume of tissue ablated. All CORT rats had cortical damage at the anterior and intermediate planes of assessment, but only 2 CORT rats had cortical damage extending as far as the posterior plane of assessment. Two CORT rats had incomplete unilateral damage to the cingulum bundle. In none of the rats was there any damage to the hippocampal formation. No rat was excluded from the CORT group.

\section{Behavior}

We here report analyses of the sensitivity, bias, hit, false-alarm, and latency measures of performance. The critical comparisons of performance were considered to be those between the performance of the SHAM group and the performance of the HIPP and, to a lesser extent, the CORT group. For the sensitivity and bias measures, the following additional within-subject factors and interactions were also examined in detail: delay effects, group $\times$ delay interactions, and group $\times$ block interactions.

On this and a similar go/no-go task utilized in this laboratory, we have found that derived measures of performance, such as sensitivity and bias, may be less reliable indices of performance than primary response and latency measures (Foster, 1989; Foster \& Rawlins, 1992). We therefore counsel caution in the interpretation of derived measures of performance. However, sensitivity and bias measures are of value in distilling large volumes of data and their use allows direct comparison with the findings of other studies (e.g., Pontecorvo, 1983; Spencer, Pontecorvo, \& Heise, 1985).

Stage 1. There was a main effect of group on bias at the $1-\sec [F(2,21)=8.26, p<.005]$ and $2.5-\mathrm{sec}$ $[F(2,21)=4.66, p<.05] \mathrm{RIs}$, with the post hoc tests 

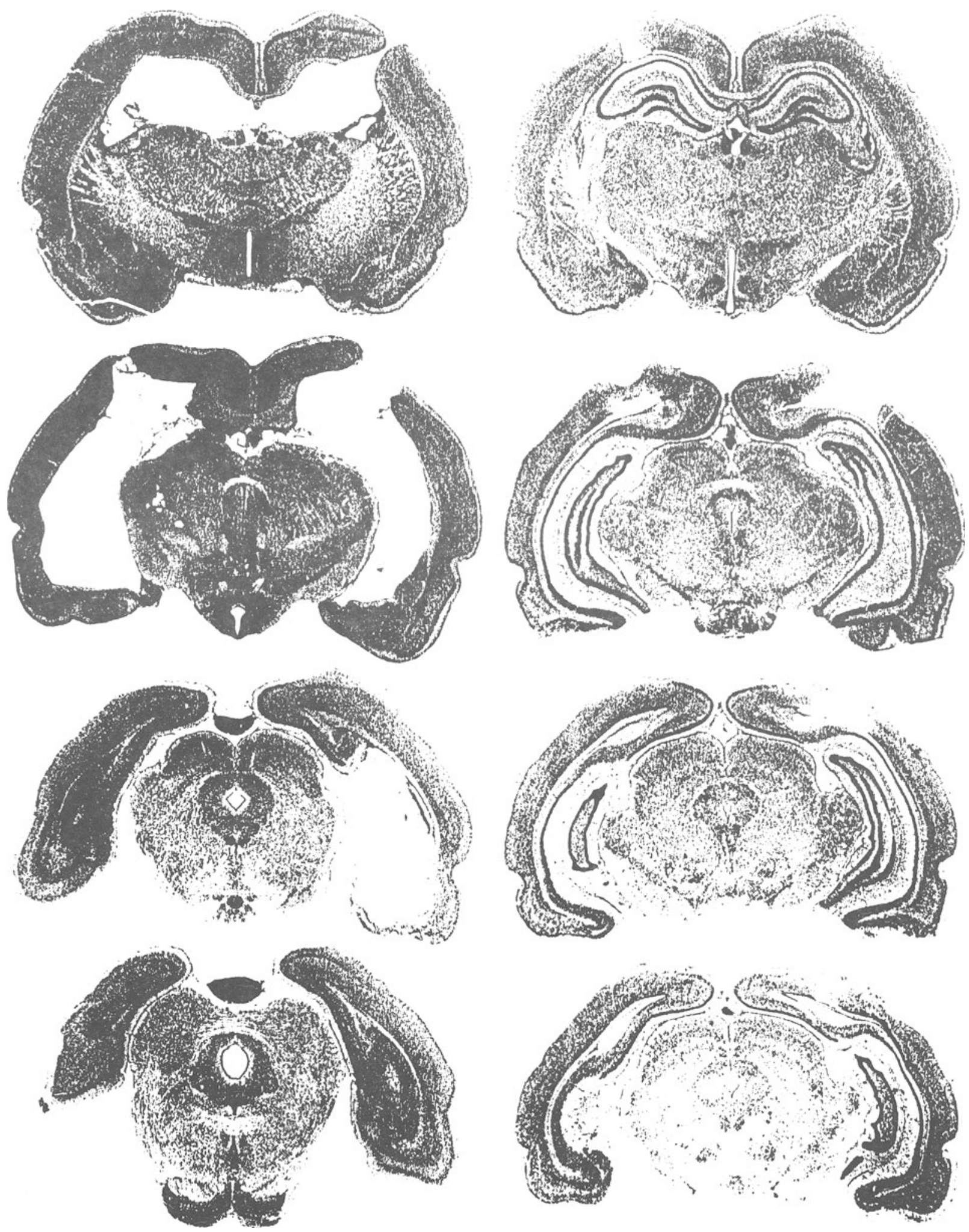

Figure 1. Photographs of coronal sections stained with Cresyl violet at the anterior (upper [first] section), intermediate (middle [second] section), and posterior (two lower [third and fourth] sections) planes of assessment. Micrographs show a rat with a sample hippocampal lesion (HIIP; left column) and a rat with a sample cortical lesion (CORT; right column). Sections correspond to plates 38 , 45 and 50 of König and Klippel's (1963) atlas. In both lesions, plate 50 of König and Klippel's atlas corresponds to the third section of the right hippocampus, fourth section of the left hippocampus. 
Table 1

Overall Means and Standard Errors of Mean in Stage 1

\begin{tabular}{|c|c|c|c|c|c|c|c|c|c|c|c|c|c|c|c|c|c|c|}
\hline & \multicolumn{6}{|c|}{$1-\sec R I$} & \multicolumn{6}{|c|}{$2.5-\sec R I$} & \multicolumn{6}{|c|}{ 10-sec RI } \\
\hline & \multicolumn{2}{|c|}{ HIPP } & \multicolumn{2}{|c|}{ CORT } & \multicolumn{2}{|c|}{ SHAM } & \multicolumn{2}{|c|}{ HIPP } & \multicolumn{2}{|c|}{ CORT } & \multicolumn{2}{|c|}{ SHAM } & \multicolumn{2}{|c|}{ HIPP } & \multicolumn{2}{|c|}{ CORT } & \multicolumn{2}{|c|}{ SHAM } \\
\hline & $M$ & $S E M$ & $M$ & SEM & $M$ & $S E M$ & $M$ & $S E M$ & $M$ & $\overline{S E M}$ & $M$ & $S E M$ & $M$ & $\overline{S E M}$ & $M$ & $\overline{S E M}$ & $M$ & $\overline{S E M}$ \\
\hline 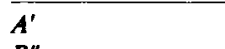 & 77 & 0.01 & 75 & 0.01 & 76 & 01 & 74 & 0.02 & 7 & 001 & .75 & .01 & 0.70 & 1 & .62 & .03 & 0.66 & 0 \\
\hline$B^{\prime \prime}$ & & & 0.66 & & & 0. & $0.88^{*}$ & & & & & & 0.70 & & & & 0.57 & .04 \\
\hline$p(\mathrm{hi}$ & 97 & 0.01 & 0.94 & 0.01 & 0.90 & 0.01 & 0.97 & 0.01 & 0.95 & 0.01 & 0.91 & 0.01 & 0.96 & 0.01 & 0.94 & 01 & 0.91 & 0.01 \\
\hline$p(\mathrm{fa}$ & 76 & 0.01 & 0.69 & 0.01 & 0.61 & 0.01 & $0.84 *$ & 0.01 & 0.77 & 0.01 & 0.68 & 0.01 & 0.92 & 0.01 & 0.88 & 0.01 & 0.81 & 0.01 \\
\hline Hit L & $1.96^{*}$ & 0.07 & 3.00 & 0.08 & 3.34 & 0.11 & $1.59 *$ & 0.07 & 2.55 & 0.09 & 2.88 & 0.11 & 1.31 & 0.07 & 2.05 & 0.08 & 2.25 & 0.12 \\
\hline fa La & $.72 *$ & 0.11 & 5.16 & 0.1 & 5.9 & 0.1 & $2.73^{*}$ & 0.10 & 4. & 0.10 & 4.90 & 0.13 & $1.64^{*}$ & 0.08 & 2.53 & 0.08 & 3.11 & 0.12 \\
\hline$p($ rfa $)$ & 0.55 & 0.01 & 0.55 & 0.01 & 0.60 & 0.01 & 0.54 & 0.01 & 0.53 & 0.01 & 0.59 & 0.01 & 0.55 & 0.01 & 0.53 & 0.01 & 0.60 & 0.01 \\
\hline
\end{tabular}

Note-RI = retention interval; HIPP = hippocampal aspiration lesion; CORT = cortical control lesion; SHAM = sham-operated control lesion; $\mathrm{fa}=$ false alarm; rfa $=$ repeat false alarm. $\quad$ *Significant between-groups differences.

revealing that bias was significantly greater for HIPP animals than for SHAMs at both intervals (Table 1).

The probability of a false alarm was greater for HIPPs than for SHAMs at the 2.5-sec $\operatorname{RI}[F(2,21)=3.67, p<$ .05]. The mean hit latency was greater for SHAMs than for HIPPs at the $1-\sec [F(2,21)=7.09, p<.005]$ and 2.5 -sec $[F(2,21)=6.25, p<.01]$ RIs. The mean falsealarm latency was greater for SHAMs than for HIPPs at the $1-\sec [F(2,21)=5.26, p<.05], 2.5-\sec [F(2,21)=$ $6.22, p<.01]$, and $10-\sec [F(2,21)=4.21, p<.05]$ RIs (Table 1).

Of the main measures of performance, there was a delay effect on sensitivity $[F(2,42)=19.17, p<.0001]$ and on bias $[F(2,42)=6.28, p<.005]$. The post hoc analysis revealed that sensitivity was inversely proportional to RI and was significantly different at all three delays. Bias was significantly greater at the $2.5-\mathrm{sec}$ RI than at the 1- and 10-sec delays (Table 1).

Stages 2-4. The results were very similar to those reported for Stage 1: HIPPs responded more vigorously than did controls on both match and nonmatch trials, but showed no reliable memory differences from the other two lesion groups.

\section{DISCUSSION}

In Stage 1 of the experiment, the maximum trial duration was $10 \mathrm{sec}$, and the RIs were of $1,2.5$, or $10 \mathrm{sec}$, with the probability of the $1-\mathrm{sec}$ interval being twice as great as that of each of the other two RIs. The results of this stage of the experiment indicate that hippocampectomy did not significantly impair performance of the basic CNM task: sensitivity was unaffected in the hippocampal group (Table 1).

Bias was greater for HIPPs than for SHAMs at both the 1- and 2.5-sec intervals, with a similar pattern of results occurring at the 10-sec RI, indicating a greater predisposition to respond, irrespective of trial type, among HIPP animals (Table 1). This tendency for responding to be somewhat unselectively disinhibited in the HIPP group was also reflected by the false-alarm and latency data (Table 1) and by the number of pretrial responses. On the latter, although between-groups differences were nonsignificant, HIPPs made a mean number of 0.37 between- trial responses, whereas CORTs made a mean of 0.1 and SHAMs made a mean of 0.14 intertrial responses.

On the main measures of performance, there was a delay effect on sensitivity, which was proportional to the length of the RI, indicating that the rats were being taxed at the longer delays (Table 1). There was no group $\times$ delay interaction, however, indicating that HIPP rats were no more impaired at longer RIs than were controls. There was also a delay effect on bias, with the rate of responding increased at the intermediate RI. This may have been due to a tradeoff, in all groups, between an increase in impulsive responding with increased delay and the initiation of more unrelated competing activities during longer between-trial intervals. However, there is some indication from the summed hit and false-alarm scores, together with the latency data, that the level and rate of responding may have increased across groups in proportion to the length of the RI, with overall responding being highest after the 10-sec interval (Table 1). This apparent discrepancy raises a concern about the validity of the nonparametric bias measure (Grier, 1971).

In light of these generally negative findings, it was decided to modify the parameters in the subsequent stages of the experiment, in order to investigate whether amendments to the basic CNM task would discriminate between different lesion groups.

In Stage 2 of the experiment, the maximum trial duration was reduced to $5 \mathrm{sec}$. This procedural change was effected in order to reduce the maximum time over which hippocampal animals were required to inhibit a "sampling" response on match trials. We reasoned that, by reducing the maximum trial duration, the probability that hippocampal animals would make responses for nonspecific reasons, such as generalized impulsivity or disinhibition, would be diminished. It was thought that such non-specific responding may have masked a more subtle mnemonic effect in Stage 1 and that, by reducing the maximum trial duration, a delay-dependent deficit would therefore be more likely to emerge.

In Stage 3 of the experiment, the maximum RI was increased to $20 \mathrm{sec}$ in order to tax further each rat's memory for the events of the previous trial. Increasing the intertrial interval is known to produce a differential impairment in the performance of hippocampal animals on 
other go/no-go and delayed alternation tasks (Rawlins, 1985; Winocur, 1985). The minimum RI was also increased from 1 to $2.5 \mathrm{sec}$ in order to minimize the likelihood that, following reinforcement, rats were failing to sample the subsequently presented stimulus because they were continuing to consume the sucrose reinforcer at the central magazine tray during the reduced duration of stimulus presentation introduced in the second stage. Although this change in procedure was not necessarily expected to differentiate between the performance of different lesion groups, it was introduced to reduce possible distortions of the data due to experimental noise.

In Stage 4 of the experiment, between-trial interference was introduced. A free dipper of sucrose was presented for a duration of $1 \mathrm{sec}$ after $0.5 \mathrm{sec}$ of each RI had elapsed. Hippocampal animals are known to be especially susceptible to the effects of interference on a variety of tasks (Jarrard, 1965; Winocur, 1979, 1982); therefore, the introduction of the intertrial interference dipper was expected to impair selectively performance in the hippocampal group.

However, despite these parametric changes in procedure, the results of Stages 2, 3, and 4 revealed a similar pattern to that shown in Stage 1, reflecting what was obviously a robust and consistent hippocampal effect. Sensitivity was unimpaired in HIPP animals, regardless of trial duration, RI, and the presence of intertrial interference. However, HIPP animals again responded more, and with a shorter latency, than did control animals on the CNM task.

These findings are thus similar to those obtained in this laboratory when hippocampal animals were tested on a similar delayed alternation unsignaled go/no-go task (Foster \& Rawlins, 1992) and would appear to be most consistent with the behavioral inhibition model of hippocampal function, rather than with either the working memory or the temporal discontiguity nonspatial theories. The predictions of these two models, each of which proposes that the hippocampus is concerned specifically with memory capacity, are inconsistent with the nonspecific behavioral effects reported here.

The results of this experiment would also seem to conflict with those obtained by Raffaele and Olton (1988) and those observed using a similar maze task in this laboratory (Rawlins, personal communication; Rawlins et al., 1991), despite the fact that there is a high level of withinsession proactive interference in both the CNM and matching-to-sample nonspatial tasks. On the matching task, deficits in response accuracy have been observed following both electrolytic lesions of the fornix-fimbria and aspiration lesions of the hippocampus identical to those performed here (Raffaele \& Olton, 1988; Rawlins, personal communication; Rawlins et al., 1991). Indeed, in the study conducted by Rawlins et al., in which animals with fornix lesions were tested together with animals with hippocampal aspiration lesions, the performance of these two groups did not differ at any stage of testing.

The discrepancy between the present findings and those reported by Raffaele and Olton (1988) may be attribut- able to procedural differences that induced rats to adopt different response strategies to perform similar tasks. Although it is not a spatial task per se, in the maze matchingto-sample task, the animal must locomote several inches to register a response to a cue located in extended space, whereas, in the CNM task, the animal responds on a lever located immediately in front of it. In the latter, the location of the cue is distanced somewhat from the location of the reinforcer, whereas, in the matching-to-sample task, the cue designates a place in the environment in which a reinforcer can be obtained.

The matching-to-sample task is also a forced two-choice recognition task, whereas, in the CNM task, only one response manipulandum is employed, with subjects' choices indicated solely by the occurrence or nonoccurrence of a response. An animal may respond or fail to register a response for a number of reasons related to level of arousal, motivation, or memory. In the presence of a readily available manipulandum, hippocampal rats may be unable to disinhibit nonspecific responding and may thus adopt a perseverative strategy. Furthermore, the intertrial intervals used in Raffaele and Olton's (1988) matchingto-sample procedure were not varied systematically. This leaves open the possibility that the impaired performance of hippocampal animals observed by Raffaele and Olton was due to a deficit in attention, perception, and/or general information-processing capacity rather than to mnemonic impairment per se.

The differences in outcome observed by Raffaele and Olton (1988) on the matching-to-sample task (hippocampal deficit) and by Aggleton et al. (1986) on the object nonmatching task (no deficit) may rest on the difference in the level of interference between the two tasks. In the former, the same two stimuli are used repeatedly throughout a test session, whereas, on the latter, target boxes are used in a trial-unique manner. It is interesting to note that, in a second study conducted by Rawlins et al. (1991; Rawlins, personal communication) using the matchingto-sample procedure but with trial-unique stimuli (similar to the design used by Aggleton et al.), neither the fornix lesion nor the hippocampal aspiration lesion produced a significant effect. It therefore seems that there may be an interaction between intrasession interference and task such that both a high degree of proactive interference and a requirement for the animal to locomote toward the cue are required to reveal a substantial hippocampal deficit. Only the matching-to-sample task using repeated stimuli satisfies both of these conditions.

A supplementary consideration is that lesion differences may contribute toward the apparent discrepancy between the findings of Raffaele and Olton (1988) and those reported here. However, as noted, Rawlins et al. (1991; Rawlins, personal communication) found a similar pattern to that observed by Raffaele and Olton on the matching-to-sample task with the same hippocampal aspiration lesions that were performed in the present study. This observation would therefore seem to indicate that procedural, rather than lesional, differences are more likely to account for the discrepancy in outcome. 
With regard to interpretation of the data reported, an important caveat concerns baseline performance levels on the CNM task. In Stage 1 of the study, the overall mean probability of a hit in the SHAM group was stable at $0.90-0.91$ over the three RIs, whereas the probability of a false alarm ranged from 0.61 to 0.81 across delays. The relatively high level of incorrect responding on match trials produced a large number of repeat match "correction" trials, such that the ratio of match to nonmatch trials was further increased beyond the 3:1 ratio of "first" match/nonmatch trials. With increasing ratios, it is possible that the task becomes less of a working memory task and more of an "oddball"-type attention task. Furthermore, because of the relatively poor overall performance of the SHAM group on match trials at the longest RI, it is possible that delay-dependent decrements in the HIPP group would yield nonsignificant effects ("floor effects"). In other words, the absence of lesion effect on sensitivity observed in the present study may not be definitive because of the limited ability of SHAM rats to discriminate trial types.

However, although these concerns are important, it should be noted that correction trials were not additional discrete trials but, rather, were continuously extended match trials in which the stimulus continued to be presented until the animal refrained from responding or until the maximum trial duration had elapsed $(10 \mathrm{sec}$ in Stage 1). Thus, if each rat responded, on average, incorrectly once on every match trial, the mean total match trial duration (first plus correction trials) would be no greater than $20 \mathrm{sec}$ in Stage 1 and $10 \mathrm{sec}$ in Stage 4. In the present study, only HIPP rats typically attained such a high level of incorrect match trial responding.

Furthermore, the means given in Table 1 refer to task acquisition and performance over a period of 36 days. Over the last 12 days of testing, as the animals reached asymptote on the task, mean sensitivity in the SHAM group varied from 0.84 to 0.72 across RIs (compared with 0.76- 0.66 over all days of testing; chance $=0.5$ ). More specifically, the mean probability of a false alarm in SHAM animals was lower over the last 12 days and ranged between 0.5 and 0.74 across the three RIs, with a corresponding reduction in the number of extended match trials. There was therefore a reduced likelihood of a floor effect occurring at the longest RI over this period of testing. HIPPs yielded scores between 0.66 and 0.90 across delays over the last 12 days of testing. Compared with those of SHAMs, these scores would seem to indicate that the increased rate of incorrect match trial responding of HIPP rats was truly nonspecific for interval duration. [In Stage 1, overall, there were indications of differences in $p(\mathrm{fa})$ at all three RIs, but these were not statistically significant at the $1-\sec (p=.08)$ and $10-\sec$ $(p=.08)$ RIs.] Furthermore, when a separate analysis was performed on the data from the last 12 days of testing, the results indicated that significant statistical betweengroup effects were limited to correct responding on nonmatch trials only, with the probability of a hit being significantly greater in HIPPs at the longest delay [HIPPs, $p($ hit $)=1-0.98$; SHAMs, $p$ (hit) $=0.92-0.91$ across delays]. These profiles of response output were largely born out in Stages 2-4 (in which, as expected, responding was generally more stable over the total period of testing than in Stage 1), thus reinforcing the interpretation that the only truly consistent lesion difference was an increase in responsivity in the HIPP group.

A general methodological issue arising out of the present study concerns the value of using signal detection theory indices to analyze performance on the CNM and similar operant choice tasks. In this experiment, a nonparametric signal detection theory analysis was used to transform the data into separate estimates of response accuracy (sensitivity) and overall tendency to respond (bias) measures. This analysis was conducted in an attempt to limit, and perhaps even eliminate, the potential confounding effects of motivational, arousal, or other nonspecific factors on the evaluation of performance.

Furthermore, this approach has the potential of alerting the experimenter to the presence of such nonspecific effects on the performance of an operant choice task. Thus, if a manipulation that decreases sensitivity also produces a large decrease in bias, one should entertain the hypothesis that the treatment decreased the level of motivation or arousal, rather than, or in addition to, causing a specific decrease in discrimination accuracy. On the other hand, if a manipulation that alters sensitivity causes little or no effect on bias, then one can relatively safely attribute the effects of the treatment to changes in discriminative processes.

While acknowledging the utility of this general theoretical approach, Sahgal (1987) argues that the theory may not always be the most appropriate means of assessing recognition memory performance and suggests alternative indices for use with two-choice operant paradigms. More specifically, in the present experiment, we noted that there were occasional discrepancies between the implications of some of the sensitivity and bias analyses and more primary measures of performance; for example, in Stage 1, bias showed a significant increase at the intermediate RI, although there was some indication from the hit, false-alarm, and latency data that response vigor increased in a delay-dependent manner. We therefore advocate prudence in the use of signal detection indices. Used judiciously, and interpreted in the context of primary values of $p(\mathrm{hit}), p(\mathrm{fa})$, and latency measures, derived signal detection scores can provide considerable experimental insight, enabling one to differentiate between response accuracy and other less specific influences on performance. However, secondary indices of performance derived from this theory should not be embraced unreservedly and without some degree of caution.

In conclusion, further experiments are required to investigate the discrepancies between the results reported here and those obtained by Raffaele and Olton (1988). These studies should utilize forced-choice two-lever operant nonmatch tasks in an attempt to simulate delayed match and nonmatch maze studies more closely. More generally, experimenters using operant-choice tasks should be 
aware of the possibility of misinterpreting findings derived from signal detection measures that have been divorced from more fundamental indices of task performance.

\section{REFERENCES}

Aggleton, J. P., Blindt, H. S., Rawlins, J. N. P. (1989). The effects of amygdaloid and combined amygdaloid-hippocampal lesions upon object recognition and spatial working memory. Behavioral Neuroscience, 103, 962-975.

Aggleton, J. P., Hunt, P. R., \& Rawlins, J. N. P. (1986). The effects of hippocampal lesions upon spatial and nonspatial tests of working memory. Behavioural Brain Research, 19, 133-146.

Douglas, R. J. (1967). The hippocampus and behavior. Psychological Bulletin, 67, 416-442.

FOSTER, J. K. (1989). The role of the cholinergic system in working memory. Unpublished doctoral dissertation, University of Oxford, Oxford.

Foster, J. K., Rawlins, J. N. P. (1992). Hippocampal aspiration lesions fail to impair performance of a delayed alternation $\mathrm{GO} / \mathrm{NO}$ GO task. Behavioural Brain Research, 47, 35-48.

GraY, J. A. (1982). The neuropsychology of anxiety: An enquiry into the functions of the septo-hippocampal system. Oxford: Oxford University Press.

GreEN, D. M., SWETS, J. A. (1966). Signal detection theory and psychophysics. New York: Wiley.

GRIER, J. (1971). Nonparametric indexes for sensitivity and bias: Computing formulas. Psychological Bulletin, 75, 424-429.

HoDos, W. (1970). A nonparametric index of response bias for use in detection and recognition experiments. Psychological Bulletin, 74, 351-354.

JARRARD, L. E. (1965). Hippocampal ablation and operant behavior in the rat. Psychonomic Science, 2, 115-116.

Kimble, D. P. (1968). Hippocampus and internal inhibition. Psychological Bulletin, 70, 285-295.

KöNIG, J. F. R. \& KIPPEL, R. A. (1963). The rat brain. Baltimore: Williams \& Wilkins.

McNicol, D. (1972). A primer of signal detection theory. London: Allen \& Unwin.

Millenson, J. R. (1971). A general language for on-line control of psychological experimentation. Behavioral Science, 16, 248-256.

Millenson, J. R. (1975). System developments in the ACT language: Towards machine independence. Behavior Research Methods \& Instrumentation, 7, 165-173.

MishkIN, M. (1982). A memory system in the monkey. Philosophical Transactions of the Royal Society of London, 298B, 85-95.

OKeEFE, J., \& NADEL, L. (1978). The hippocampus as a cognitive map. Oxford: Oxford University Press.

Olton, D. S., Becker, J. T., \& Handelmann, G. E. (1979). Hippocampus, spece, and memory. Behavioral \& Brain Sciences, 2, 313-365.

Pollack, I., \&orman, D. A. (1964). A nonparametric analysis of recognition experiments. Psychonomic Science, 1, 125-126.

PONTRCORvo, M. J. (1983). Effects of proactive interference on rats' continuous nonmatching-to-sample performance. Animal Learning \& Behavior, 11, 356-366.

RAffaele, K. C., Olton, D. S. (1988). Hippocampal and amygdaloid involvement in working memory for nonspatial stimuli. Behavioral Neuroscience, 102, 349-355.
Rawuins, J. N. P. (1985). Associations across time: The hippocampus as a temporary memory store. Behavioral \& Brain Sciences, 8 , 479-528.

RAwuns, J. N. P., BenNett, R. C. (1980). A headholder for visually guided surgery in rats. Physiology \& Behavior, 24, 415-416.

Rawlins, J. N. P., Feldon, J., \& Gray, J. A. (1980). The effects of hippocampectomy and of fimbria section upon the pertial reinforcement extinction effect in rats. Experimental Brain Research, 38, 273-283.

Rawlins, J. N. P., Lyford, G., Seferiades, A. (1991). Does it still make sense to develop nonspatial theories of hippocampal function? Hippocampus, 1, 283-286.

SAHGAL, A. (1987). Some limitations of indices derived from signal detection theory: Evaluation of an alternative index for measuring bias in memory tasks. Psychopharmacology, 91, 517-520.

Spencer, D. G., Pontecorvo, M. J., \& Heise, G. A. (1985). Central cholinergic involvement in working memory: Effects of scopolamine on continuous nonmatching and discrimination performance in the rat. Behavioral Neuroscience, 99, 1049-1065.

SQUiRE, L. R., Zola-Morgan, S. (1991). The medial temporal lobe memory system. Science, 253, 1380-1386.

WinOCUR, G. (1979). Effects of interference on discrimination learning and recall by rats with hippocampal lesions. Physiology \& Behavior, 22, 339-345.

WINOCUR, G. (1982). Radial-arm-maze behavior by rats with dorsal hippocampal lesions: Effects of cueing. Joumal of Comparative \& Physiological Psychology, 96, 155-169.

WiNocUR, G. (1985). The hippocampus and thalamus: Their roles in short- and long-term memory and the effects of interference. Behavioural Brain Research, 16, 135-152.

\section{APPENDIX \\ SDT Measures}

Assumptions required for parametric signal detection theory indices ( $d^{\prime}$ and $B^{\prime}$; Green \& Swets, 1966; McNicol, 1972) could not be validated in the present study. The data were therefore transformed into nonparametric indices of sensitivity $\left(A^{\prime} ;\right.$ Pollack \& Norman, 1964) and bias ( $B^{\prime \prime}$; Grier, 1971; Hodos, 1970), calculated according to the formulas given below. $A^{\prime}$ and/or $B^{n}$ can assume infinite values when $p(\mathrm{fa})=1$ and/or $p($ hit $)=0$, leading to a significant number of missing data points-an issue that, to our knowledge, has not been commented upon in the extant literature. One approach to this problem, which we chose to adopt here, is to modify scores of $A^{\prime}$ and $B^{n}$, which assume these values: problematic values of $p(f a)$ and $p$ (hit) were adjusted to values of 0.99 and 0.01 , respectively. These modifications were, however, not made in calculating the primary $p$ (hit) and $p(\mathrm{fa})$ scores.

$$
\begin{gathered}
A^{\prime}=0.5+\frac{[p(\text { hit })-p(\mathrm{fa})]+[p(\mathrm{hit})-p(\mathrm{fa})]^{2}}{4 \cdot p(\mathrm{hit}) \cdot[1-p(\mathrm{fa})]} \\
B^{\prime \prime}=\frac{\left[p(\text { hit })-p(\text { hit })^{2}\right]-\left[p(\mathrm{fa})-p(\mathrm{fa})^{2}\right]}{\left[p(\text { hit })-p(\mathrm{hit})^{2}\right]+\left[p(\mathrm{fa})-p(\mathrm{fa})^{2}\right]} .
\end{gathered}
$$

(Manuscript received September 17, 1991 revision accepted for publication April 7, 1992.) 